\title{
A PRELIMINARY REPORT ON THE PRODUCTION, ACTION AND THERAPEUTIC EFFECTS OF LEUKOCYTIC EXTRACTS*
}

\author{
R. A. ARCHIBALD AND GERTRUDE MOORE \\ OAKLAND, CAL.
}

Subsequent to the publication of an article by Hans Zinsser, George W. McCoy and C. W. Chapin ${ }^{1}$ on The Protective Influence of Leukocytic Substances on Experimental Plague, one of us conceived the idea that if leukocytic substances could be obtained in sufficient quantities to be not only therapeutically practical, but commercially possible, a decided step forward would be made in the biological treatment of infectious diseases.

Prior to the publication of the above mentioned article, Hiss and Zinsser $^{2}$ demonstrated in an experimental way that leukocytic extracts could be prepared by injecting into the pleural cavities of rabbits 10 c.c. of a meat extract broth containing 3 per cent. starch and 5 per cent. aleuronat. At the end of twenty-four hours a copious, and very cellular exudate will have accumulated in the pleural cavities. This is obtained by killing the rabbits, and removing the exudate which is immediately centrifuged. The leukocytic sediment is diluted with twenty volumes of sterile distilled water to one volume of sediment, and autodigestion is permitted to take place in the incubator, following which the extract is stored in a refrigerator until used.

In experimenting with animals, Hiss and Zinsser observed that pneumococcus, staphylococcus, streptococcus, meningococcus, typhoid, dysentery, and cholera infections in rabbits and guinea-pigs, were profoundly modified when injections of leukocytic extracts prepared as above described were administered intraperitoneally or subcutaneously. during the course of the infection. In many cases, animals were saved by these substances from infections which proved rapidly fatal in untreated control animals, even when the protective injections were made as late as twenty-four hours after intravenous infections.

* From the Research Department of the Western Laboratories, Oakland, Cal.

* Read before the Medical Research Society of the East Bay Counties, Oakland, Cal., April 4, 1914.

* Submitted for publication, April 30, 1914.

1. Zinsser, Hans, McCoy, Geo. W., and Chapin, C. W.: The Protective Influence of Leukocytic Substances on Experimental Plague, Jour. Med. Research, 1911, xxiv, No. 3.

2. Hiss, P. H., and Zinsser, Hans: Jour. Med. Research, 1908, xiv, No. 3 ; 1909, xv, No. 3. 
In applying this method of treatment by subcutaneous injections to infections in man, Hiss and Zinsser observed distinctly beneficial results in cases of epidemic cerebrospinal meningitis, lobar pneumonia, staphylococcus infections, and erysipelas.

Hiss and Zinsser's idea was that injecting into infected subjects the substances composing the cells usually found in exudates in a diffusible form, such substances would, following absorption, relieve or stimulate the fatigued leukocytes.

Later, Zinsser, assisted by McCoy and Chapin, conducted a series of experiments on white rats with a view to ascertaining whether substances obtained from normal leukocytes would modify in any way the course of experimental infection with the bacillus pestis. As the results of their experiments these workers found that the use of emulsions of normal leukocytic substances obtained from rabbits exerted a distinctly protective influence, even when such substances were administered twenty-four hours after the inoculation of rabbits with virulent plague organisms.

While the results obtained by the above mentioned investigators were intensely interesting from a practical point of view their methö of obtaining leukocytic substances must be considred as of little value, since the injection of a protein substance into the pleural cavities of rabbits yields such a small amount of leukocytic substance as to rob it of any value therapeutically.

Furthermore, the leukocytes so obtained are, strictly speaking, inflammatory letkocytes, which have probably given up much of their potential qualities, or in other words, have become fatigued or exhausted with the task of caring for the foreign protein, the injection of which caused them to migrate into the pleural cavity.

For the purpose of throwing some light on the nature of bactericidal substances in leukocytic extracts, Wilfred H. Manwaring ${ }^{3}$ conducted a series of experiments. Manwaring started out with the assumption that there exists powerful bacteriolytic substances within the cytoplasm of certain body cells, and that this fact is amply demonstrated in the automatic sterilization of old abscess cavities and the sterilizing of the pneumonic lung, which is assumed to be due to the liberation of endolysins, the result of cellular disintegration. His work was done with a view of extending the present knowledge of these lytic substances, and to determine the approximate chemical nature of the bacteriolytic substances that might be extracted from leukocytes.

3. Manwaring, Wilfred H.: The Nature of the Bactericidal Substances in Leukocytic Extract, Jour. Exper. Med., 1912, xvi, No. 3. 
In obtaining leukocytes for these experiments, Manwaring at first followed the practice of injecting a 5 per cent. suspension of aleuronat into the pleural cavities of rabbits, but he found that he was decidedly handicapped in his study of the nature of the extracts so obtained on account of the small amount of substance available. By injecting aleuronat into the pleural cavity of a rabbit, one seldom secured more than a single cubic centimeter of leukocytes. In order to overcome this obstacle, he selected the horse for the continuation of his work. $\mathrm{His}$ treatment consisted in injecting into the pleural cavity of a horse, from 300 to 500 c.c. of a 5 per cent. aleuronat in 2 per cent. starchpaste solution, the starch being added to aid in holding the aleuronat in suspension. Following such an injection there is usually found from 1 to 3 liters of pleural exudate daily for the first week; the daily yield then decreases, usually ceasing entirely about the fifteenth day. The exudate contains about 5 per cent. of leukocytes.

Manwaring, while not being able to arrive at a final conclusion as to the nature of the bactericidal substances in leukocytic extracts, did reach the logical conclusion, as the results of his investigations, that the bactericidal substance present is probably an enzyme.

We could, did circumstances permit, go on and recount the results obtained by other investigators, showing that leukocytes do contain intracellular substances which possess proteolytic properties, but from a study of the question from a therapeutic standpoint, the results obtained by later investigators, and our own personal work, indicate that the direct bactericidal action brought about by the parenteral injection of leukocytic substances does not influence to a marked degree the progress of an infectious disease. While able to demonstrate in vitro bactericidal properties in leukocytic extracts produced by the method devised by us, it was long ago concluded that such bactericidal powers were not sufficient to account for the profound changes actually produced in animals suffering from infectious diseases while undergoing a course of treatment by the parenteral injection of leukocytic substances. For the reason of these profound changes we must look elsewhere than to the direct lytic action on the invading bacteria.

It is a well-established fact that the parenteral injection of protein substances increases the leukocytic count, and even the injection of foreign vegetable proteins such as nuclein produce, in many infections, marked beneficial effects. It would seem, however, in carrying out leukocytotherapy, that in order to produce a hyperleukocytosis without unnecessary strain on the organism, it is eminently more logical for the organism to care for parenteral injections of substances similar to its own elements than to any elements which are of an alien character. 
It is also a well established fact that in the treatment of diseases, any method which will stimulate the leukocyte-producing organs to a more active function, thereby producing a leukocytosis, is and must of necessity be of positive bennefit. The great problem before us then in this connection is to ascertain the simplest and most innocent means of accomplishing this end.

About the beginning of the year 1911 a series of experiments were instituted with a view of obtaining leukocytic substances in quantities that would render them not only therapeutically practical, but also commercially possible. The general plan adopted was to obtain leukocytes directly from the blood of normal animals, avoiding as far as possible any changes in their character during the process. It is unnecessary to go into detail as to the various methods employed to bring this about. Suffice it to say that after much experimental work, the following general technic was adopted:

\section{TECH NIC}

The blood is obtained under aseptic precautions from the jugular vein of the horse, dog, or other domestic animal; is allowed to flow into sterile flasks containing a sufficient quantity of 1 per cent. sodium citrate solution to prevent coagulation. When the blood arrives at the laboratory, sodium citrate solution is added in varying amounts, depending on the species of animal from which the blood was obtained; to this is added five-tenths of 1 per cent. acetic acid, the necessary amount of which also depends on the character of blood being treated. The mixture is then centrifuged in a high powered centrifuge and the supernatant fluid is discarded. The sediment, which is very rich in leukocytes, is washed several times with physiological salt solution, ground with quartz sand and neutralized. To the resulting sediment distilled water is added in the proportion of about four volumes of water to one of sediment. This mixture is exposed to a temperature of $58 \mathrm{C}$. for one hour, after which it is placed in the incubator until digestion of the leukocytes is complete. It is finally centrifuged, the supernatant fluid decanted and sufficient trikresol added for preservative purposes. Each lot prepared as described is carefully examined bacteriologically and its ability to produce certain definite blood changes is determined by animal inoculation.

While a great many experiments have been carried out, not only on different animals, but also on human beings, for the purpose of shedding some light on the action brought about by the parenteral injection of leukocytic extracts, it is obviously unnecessary and impracticable to submit the great mass of data so obtained at this time. We wish, however, to present the results of a few experiments which will suffice to demonstrate some of the blood changes that follow injections of the extracts.

\section{EXPERIMENTS}

Experiment 1.-For the purpose of ascertaining the different effects, if any, following parenteral injections of leukocytic substances obtained from various animals into a series of animals of the same species, with a view of determining 
whether the extracts of a different species will give rise to the same changes as leukocytic extracts from the same species, the following experiment was instituted:

Four guinea-pigs of equal weight were injected as follows: Pig 1 received 1 c.c. of horse leukocytic extract; Pig 2, 1 c.c. of dog leukocytic extract; Pig 3, 1 c.c. of guinea-pig leukocytic extract, and Pig 4, 2.5 c.c. nuclein (P. D.).

Chart 1 shows their total leukocytic curves and the total polymorphonuclear counts.

It will be observed that the injection of leukocytic extracts obtained from three different sources as well as the nuclein produced a marked leukocytosis. The leukocytic substances, with the exception of the guinea-pig extract, produced a greater reaction than the nuclein.

The dog extract produced the most marked reaction, the total leukocytic increase amounting to about 300 per cent. The differential

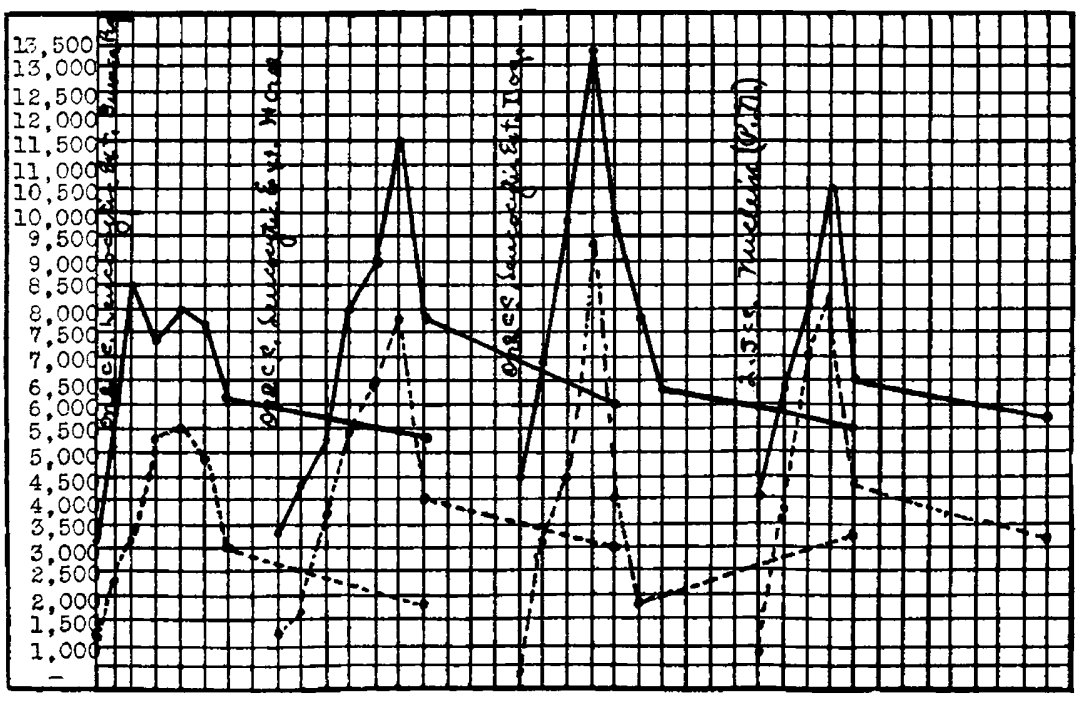

Chart 1.-Guinea-pig series. Each square represents a one and one-half hour interval. In this and following charts the solid line indicates total white count; line of short dashes, total polynuclear count; dotted line, total neutrophil count; dash and dot line (-.......), total eosinophil count.

counts showed that the dog leukocytic extract caused the most profound changes, the neutrophil increase amounting to 350 per cent., and the eosinophil count increasing from 3 to 14 per cent. The large mononuclears showed, in many cases, bundles of chromatin in their protoplasm. The nuclei of these cells were crowded to one side with a loose net-work of chromatin in the protoplasm on the opposite side. These cells appeared about one and one-half hours after the injection; gradually increasing in number for about six hours, when about onethird of the cells showed this change. In the case of the guinea-pig injected with guinea-pig leukocytic extracts, the polymorphonuclear 
leukocytes showed practically no granules and no red staining before the injection was made. A few red granules were noticed at the second count and at the third count, with but few exceptions, all the neutrophils showed distinct red granules in their protoplasm. These granules decreased in size and number until they had practically disappeared at the twenty-fourth hour after injection.

In the guinea-pig injected with horse leukocytic extract, the polymorphonuclear leukocytes showed no red granules before injection, but at the second count the red granules were at their height, disappearing at the third count, a few reappearing on the following day.

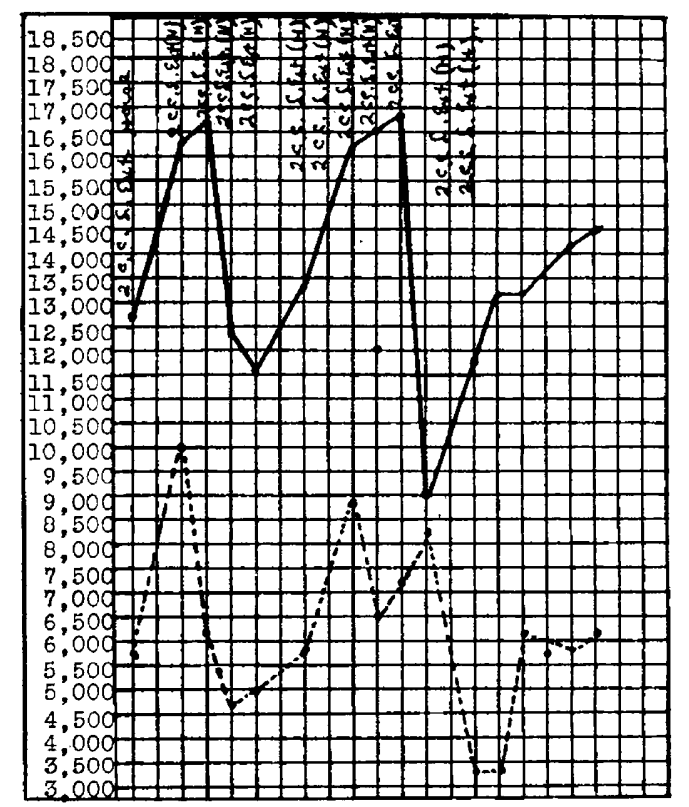

Chart 2.-Rabbit 1. Each square represents twenty-four-hour interval.

No counts could be made from the nuclein-treated pig until four and one-half hours after the injection, due to the fact that the blood clotted so rapidly. At this time a few red granules were observed in a few of the cells, but this phenomenon was not nearly so marked as in the animals injected with the leukocytic extracts.

Experiment 2.-Chart 2 shows the daily blood picture of Rabbit 1, which received twelve subcutaneous injections of 2 c.c. horse leukocytic extract at twenty-four and forty-eight-hour intervals. Blood-counts were taken daily, commencing at the time of the first injection, and continued for fifteen days. Smears made at the time of the first injection showed the protoplasm of the neutrophils, with a few exceptions, to be free from granules, the granules in these exceptions being very fine and taking a distinct red stain. At no time 
after the injections of the extract were the granules as fine, nor as few in number as before. Practically all the polymorphonuclear cells contained large, distinct, red granules; these granules had not decreased in number seventytwo hours after the last injection, at which time observations ceased.

It will be observed that marked total leukocytic curves occurred throughout the experiment. Differential counts showed that the increase in the eosinophil count and decrease of the neutrophil count, and the increase of the neutrophil count and the decrease of the

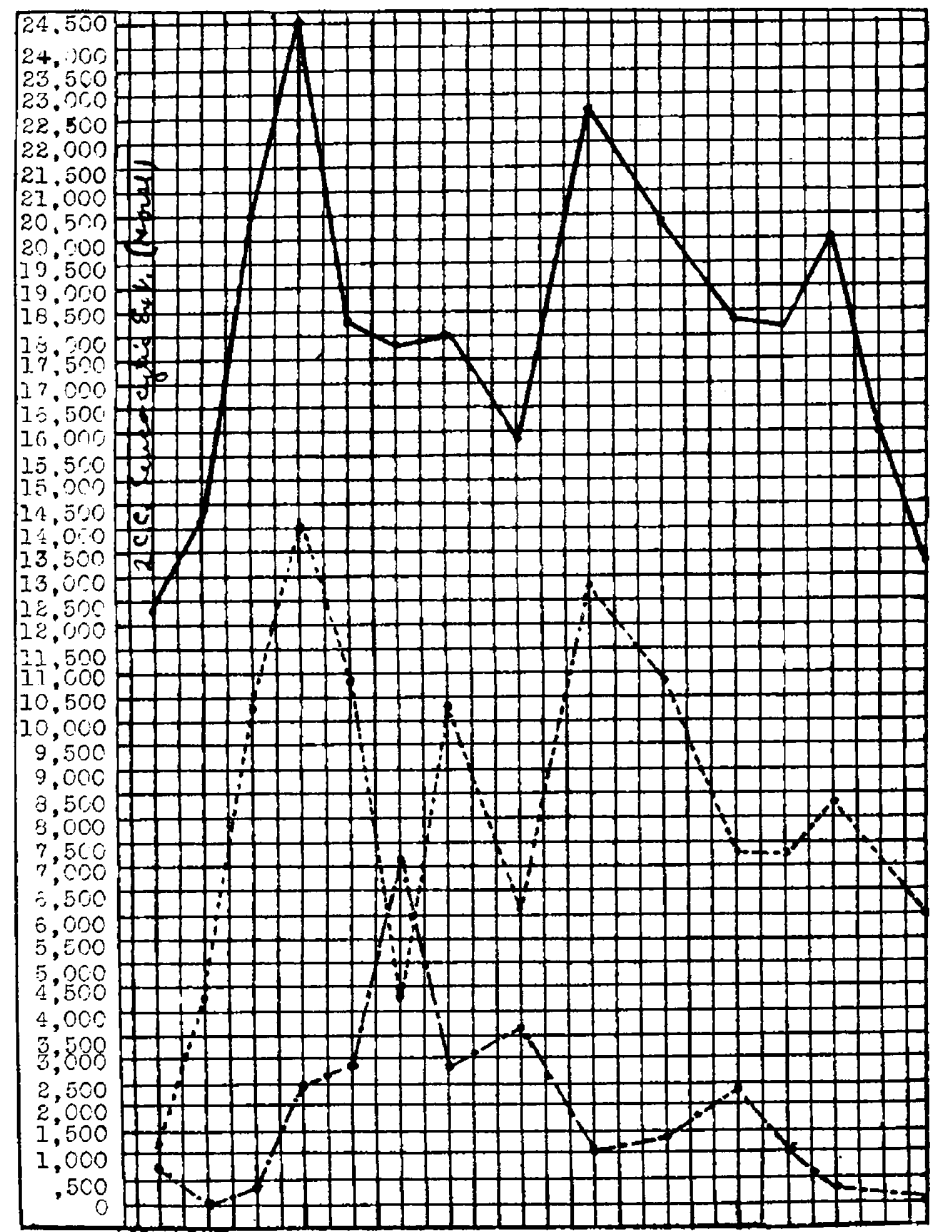

Chart 3.-Rabbit 2. Each square represents a one-hour-interval.

eosinophils kept pace with the rise and fall of the total leukocytic count. When the leukocytes took an upward curve the eosinophils also increased, reaching at one time a height of 45 per cent.

Experiment 3.--Rabbit 2 was given a single injection of 2 c.c. horse leukocytic extract. Blood-counts, as shown in Chart 3, were taken at two-hour intervals for thirty-six hours. The neutrophils showed much the same normal 
picture as in Rabbit 1. The red granules began to appear four hours after injection, and increased to a maximum in size and number between the fourth and sixth hours. These granules did not disappear during the time of the experiment, but decreased in size and number from the tenth hour on. The total count showed a drop during the first two hours, and then took an upward curve reaching the maximum height of 100 per cent. above the normal about the sixth hour. The differential counts showed an increase and decrease of the eosinophils and neutrophils coincident with the curves formed by the total leukocytic counts. The total neutrophil count at its maximum reached a point of 700 per cent. above the normal and the eosinophil total count 1,100 per cent. above the normal.

Experiment 4.-With a view of demonstrating the effects of leukocytic extracts on a human being the following experiment was carried out:

Chart 4 shows the blood pictures of a man subsequent to a subcutaneous injection of 2 c.c. of dog leukocytic extract.

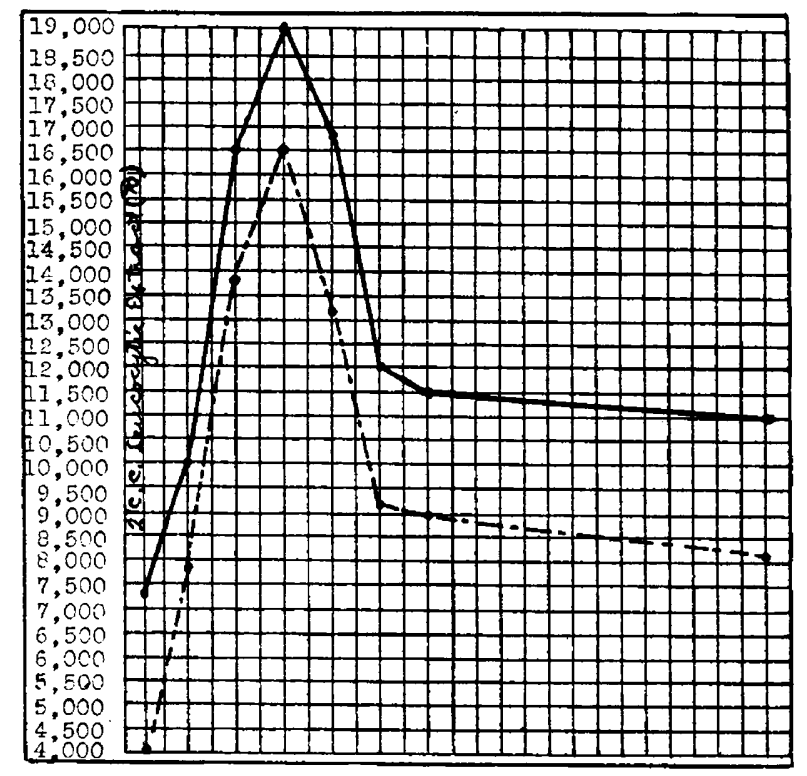

Chart 4.-Normal man. Each square represents a one-hour-interval.

A white and differential count was made just prior to the injection and subsequent counts were made at two-hour intervals for a period of twelve hours. A final count was made twenty-six hours after the injection.

The curve showed a continual increase beginning two hours after the leukocytic extract was administered, reaching its maximum at about the sixth hour. At this point the total leukocytic count was slightly over 150 per cent. above the normal and the polymorphonuclears had increased to about 500 per cent. From the sixth to the twelf th hours there was a gradual decrease both of the total leukocytic and polymorphonuclear counts. Twenty-six hours after the injection, the count had not changed from that found at the twelfth hour. At this time the total leukocytic count was about 50 per cent. above the normal, with a polymorphonuclear count a little over 100 per cent. above the normal.

The therapeutic application of leukocytic extracts to humans and animals has been followed out during the past three years. Various intiections have been treated, in some types, more especially acute 
infections, with most gratifying results, while in others, especially old chronic infections, the beneficial results were not so marked. Some of the diseases of human beings to which the treatment has been applied, and which have yielded the most pleasing results, are pneumonia, puerperal sepsis, erysipelas, empyema, bacteremia, acute pyemia, gonococcus infection, rheumatism, etc.

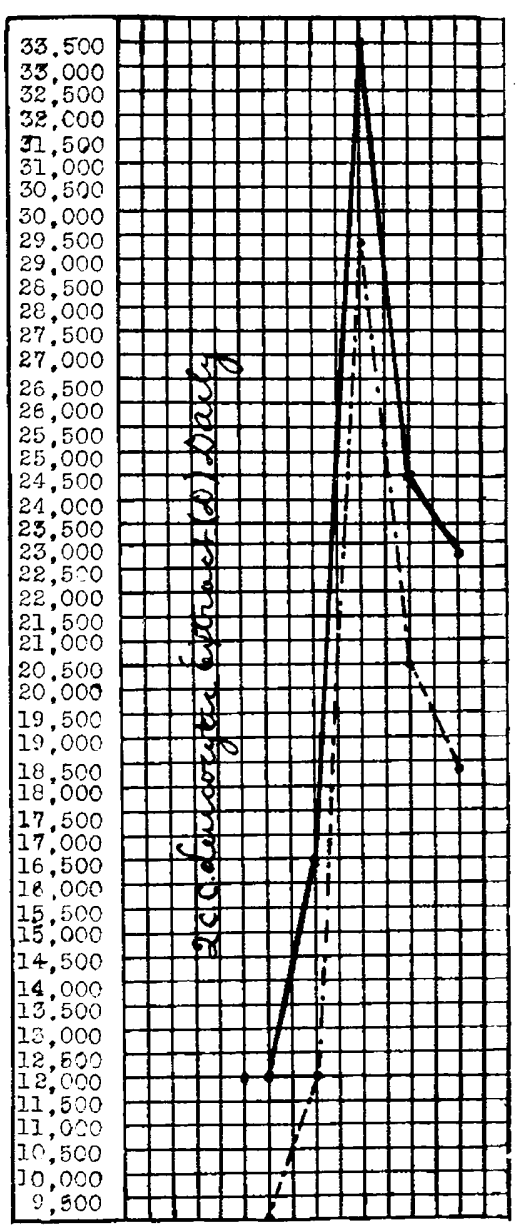

Chart 5.-Lobar pncumonia (left lower lobe). Each square represents a twenty-four-hour interval.

The following are a few of the records selected from a number of human cases treated with leukocytic extracts:

\section{CASE REPORTS}

CASE 1 (Chart 5. Lobar Pneumonia).-Miss M.. a Portugese school-girl aged 16, was first seen at 2 p. m., March 10, at which time she gave a history of a severe chill twenty-four hours previously. This was followed by fever, 
headache, nausea, vomiting, and pain in the left chest. Examination showed a temperature of $103.6 \mathrm{~F}$., pulse 112 , respiration 36 , white blood-count 12,000 , polymorphonuclear cells 70 per cent. with a dull area over the lower lobe of the left lung. Two cubic centimeters of leukocytic extract was given once daily for eight days. Following the first dose of extract the white blood-count rose to 33,400 with 88 per cent. polymorphonuclear cells, and the total count remained above 23,000 during the remainder of her illness. After the third dose the temperature came down by lysis, reaching normal on the eleventh day of the disease.

CASE 2 (Chart 6 . Cellulitis).-The patient, a young man, when first seen complained of a swelling, redness, and pain in the right arm and adjacent chest wall. The constitutional symptoms of nausea, vomiting and headache were severe, the temperature was $103 \mathrm{~F}$. and there was no tendency to localization or pus formation. At the time of admission to the hospital the white blood-count was 25,000 ; unfortunately no differential was made.

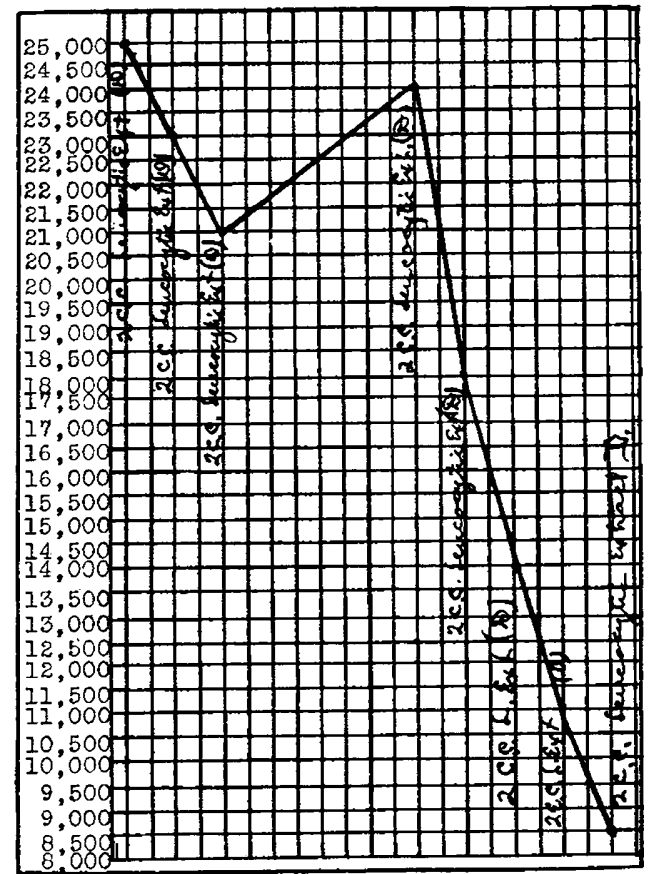

Chart 6.-Cellulitis. Each square represents a twenty-four-hour interval.

The treatment at this time consisted in the subcutaneous injection of 2 c.c. of leukocytic extract once daily, with the exception of the second dose, which followed the first after a period of twelve hours. Both the local and general symptoms were very much improved after the second dose and on the third day the temperature was normal, the patient felt well except for a slight tenderness in the affected part, the skin was normal in color, but there still remained some swelling. On account of the improved condition, it was thought best to discontinue the use of the extract at this time. That the treatment should have been continued longer was evidenced by the fact that on the seventh day, which was the fourth day without treatment, all the local and general symptoms returned, following a sudden and severe chill. The temperature again 
reached $103 \mathrm{~F}$. and the total leukocyte count 24,000. Two cubic centimeters leukocytic extract were again given once daily for the next six days. The general symptoms as before, quickly improved, and following the second dose the infection became localized and an abscess formed which was drained surgically. Healing was rapid and on the twelfth day after admission the patient left the hospital well. The chart accompanying this record is most interesting. It will be noticed that the high blood-count on admission decreased after the first doses of the extract, and increased when the extract was discontinued, to again decrease as soon as the treatment with the extract was resumed. As no blood-counts were made on the fourth, fifth and sixth days, the lowest bloodcount reached following the first doses of extract is not known.

CaSe 3 (Puerperal Sepsis).-On the third day following confinement Mrs. G. developed a temperature of $104 \mathrm{~F}$. with nausea, vomiting and intense headache. On the following day she had several slight chills, with temperature continuing high, and accompanied by some delirium and later stupor. The patient was then removed to the hospital and 2 c.c. leukocytic extract given daily. The following day she was brighter, said she felt better, temperature dropped to $102 \mathrm{~F}$. There was no further delirium, improvement continued, and the temperature reached normal four days after the administration of the extract. A blood culture made at the time this patient entered the hospital showed the presence of the Staphylococcus aureus.

Case 4 (Empyema).--Mr. B. injured by a fall from a telephone pole Oct. 17,1913 , was removed to the hospital, Oct. 22, when an abdominal operation was performed for the relief of a general peritonitis. Later an empyema developed which was operated on November 20 . The temperature, which had ranged from 102 to $104 \mathrm{~F}$. up to this time, fell to normal following the removal of the pus from the pleural cavity, but the pyothorax quickly returned and the temperature again rose, necessitating a second aspiration. A glycerin-formaldehyd solution was introduced after each aspiration. Up to December 13, when the daily subcutaneous injections of leukocytic extract were commenced, the temperature had ranged between 101 and $102 \mathrm{~F}$., except for brief intervals following drainage. From this time on, however, the temperature fell slowly, reaching the normal on December 28, when the patient reported feeling well and on December 30 left the hospital with no signs of empyema.

CASE 5 (Facial Erysipelas).-Mr. S., aged 60, when first seen presented an erysipelas of three days' duration involving the entire face, neck and both ears. $\mathrm{His}$ temperature at this time was $105 \mathrm{~F}$. The treatment consisted of daily subcutaneous injections of 2 c.c. leukocytic extract. After the second dose the inflammation ceased to spread, the affected area became lighter in color, and the temperature fell to normal. On the twelfth day after the onset the patient returned to his work, while in previous attacks he had been confined to his bed for from eighteen to twenty-one days.

Some interesting data concerning "Clinical Studies on the Curative Action of Leukocytic Extracts in Infective Processes" by Hiss and Dwyer, ${ }^{4}$ are worthy of note in this connection. In this article the results obtained by the use of leukocytic extracts on a number of cases of erysipelas are tabulated.

Total number of cases treated, 148.

Recovered, 143 ; mortality $=3.38$ per cent.

General average duration of treatment, 3.18 days.

Number of recoveries of patients under 1 year old, 8 out of 12 .

Percentage of recoveries of patients under 1 year old, 66.6 per cent.

Mortality among patients over one year, 0.73 per cent.

4. Hiss and Dwyer: Clinical Studies on the Curative Action of Leukocytic Extracts in Infective Processes, New York Med. Rec., 1913, 1xxxiv, No. 11. 
These authors also refer to the results obtained by Dr. Adrian Lambert who treated fifty cases of erysipelas at the Bellevue Hospital and obtained 50 per cent. recoveries of babies under one year. Dr. Lambert concluded from his experience that leukocytic extracts will abort infections which are treated within twenty-four hours. Older cases are ameliorated and may abruptly terminate. Toxic symptoms, delirium, headache, nausea, vomiting and pain are relieved. Rash does not disappear immediately, but is apt to localize.

It is fully realized that these therapeutic agents are at present undergoing the "acid test" and it is difficult to prevail on the therapeutist to give them a trial, unless as a last resort. Hence the list of infections for which we can conscientiously recommend the treatment is more limited than it should be. It is believed, however, from our experience in the treatment of animal diseases, that leukocytic extracts have a wide range of therapeutic application and that their use is warranted in all acute infections at least.

In animals the treatment has been applied to a great variety of local and general infections; such diseases of the horse as pneumonia, purpura, influenza, laminitis, strangles, and many local infections have yielded more rapidly to the use of the extracts than to any other treatment heretofore applied. In such diseases of the dog and cat as pneumonia, so-called distemper, and the very common bowel infections in these small pet animals, the results have been most gratifying.

In some of the above mentioned diseases in animals the agent seems to act as a specific, if we may be permitted to use the term. Apparently hopeless infections, even those in which the mortality is ordinarily very high, make good recoveries following daily injections of the extracts.

In such cases as purpura in the horse and distemper in the dog; where the treatment is commenced before the animals have lost their reactive powers, about the third day following the daily injections of the extracts rapid improvement in the clinical manifestations are observed, and this improvement continues until recovery is complete: provided the treatment is kept up until immunity has been well established.

It has been invariably observed that the first and second injections of leukocytic extracts have the tendency slightly to aggravate the toxic symptoms, even to the extent of producing slight uneasiness on the part of the affected animal, but these conditions are soon followed by improvement. These observations would seem to demonstrate that the initial effect of the extract is to increase intoxication by the liberation of toxic elements, which, providing the individual is capable of react- 
ing, stimulate the machinery of immunization and the consequent production of immune elements in sufficient quantities to control or overcome the infection.

We fully realize that there are a great many problems incidental to the production, nature, and effects of leukocytic substances that require solution, but we believe that what has thus far been accomplished may prove to be of some assistance in future study and investigation. We also believe that the facts already obtained warrant the following conclusions:

\section{CONCLUSIONS}

1. Leukocytic substances possessing positive therapeutic value may be procured from leukocytes obtained directly from the blood of normal animals.

2. Leukocytic substances may be obtained directly from the blood of normal animals in quantities which render them not only therapeutically practical but commercially possible, conditions that can hardly be claimed for leukocytic extracts prepared from inflammatory leukocytes.

3. The parenteral injection of leukocytic substances causes profound changes in the blood, producing a marked leukocytosis and marked changes in the individual leukocytes. As to the significance of these phenomena no opinion can be offered at this time.

4. Leukocytic extracts have not sufficient direct bactericidal powers to account for the profound changes produced following their parenteral introduction into an animal suffering from an infectious disease, and the beneficial results obtained are probably due to the fact that the extracts furnish elements which stimulate the fatigued or exhausted leukocytes, enabling them to carry on their function of phagocytosis and produce the changes incidental thereto.

181 Monticeto Avenue-Bauer Apartments. 